Estudios Románicos, Volumen 28, 2019, pp. 317-329

ISSN: 0210-491

eISSN: 1989-614X

DOI: https://doi.org/10.6018/ER/376921

\title{
DÉSORIENTALE DE NÉGAR DJAVADI : EXEMPLE D'ÉCRITURE AUTOFICTIONNELLE COMME TECHNIQUE DISCURSIVE RÉCURRENTE DANS LES LITTÉRATURES FRANCOPHONES CONTEMPORAINES
}

(Désorientale by Négar Djavadi : an example of autofiction writing as a common discursive technique in contemporary francophone literatures)

\author{
Beatriz Mangada Cañas* \\ Universidad Autónoma de Madrid
}

\begin{abstract}
The main objective of this article is to illustrate the preponderance of autofictional writing in the current Francophone literary scene. We wil analyze Désorientale, the first novel by the Iranian writer Négar Djavadi, who writes in French. The study of her production context will show the relevance of her inclusion in the current paradigm of writers of very different origins who have chosen French as a means of literary expression to give voice to their testimony as women and / or as exiles. The use of autofictional writing becomes a narrative technique common to all of them.
\end{abstract}

Keywords : Négar Djavadi ; Autofiction ; Francophone world ; Literature ; Exil ; Testimony.

Resumen : Este artículo se propone como principal objetivo ilustrar la preponderancia de la escritura autoficcional en el panorama literario francófono actual mediante el análisis crítico de Désorientale, primera novela de la escritora francófona de origen iraní, Négar Djavadi. El estudio de su contexto de producción permitirá mostrar la pertinencia de su inclusión en el paradigma actual de escritoras de orígnenes muy diversos que han elegido el francés como medio de expresión literaria para dar voz a su testimonio como mujeres y/o como exiliadas. El recurso a la escritura autoficcional se convierte en una técnica narrativa común a todas ellas.

${ }^{*}$ Dirección para correspondencia: Beatriz Mangada Cañas. Departamento de Filologías y su didáctica. Facultad de Formación de Profesorado y Educación. Universidad Autónoma de Madrid. Campus de Cantoblanco. 28049 Madrid. (beatriz.mangada@uam.es) 
Palabras claves : Négar Djavadi ; Autoficción ; Francofonía ; Literatura ; Exilio ; Testimonio.

\section{Les écritures autofictionnelles dans le panorama littéraire francophone actuel}

Dans le panorama littéraire francophone actuel, les noms d'écrivains d'origines diverses qui ont choisi le français comme langue d'expression littéraire sont nombreux. En 2005, Anne-Rosine Delbart leur consacrait un ouvrage intitulé Les exilés du langage. Un siècle d'écrivains français venus d'ailleurs (1919-2000), où elle dressait un inventaire détaillé d'auteurs bien divers qu'elle proposait de classer en fonction de deux grandes catégories : les sédentaires nés en terres francophones et les nomades. L'étude se complétait avec une approche des axes thématiques partagés, des enjeux du langage mis en récit et des mécanismes d'énonciation autour des discours fragmentés et des polyphonies inhérentes à l'écriture d'un moi hybride. Un inventaire descriptif qui trouvera une amplification en 2012 dans le classique dirigé par Mathis Moser et Mertz-Baumgartner, Passages et ancrages. Dictionnaire des écrivains migrants de langue française (1981-2011).

Un an avant, en 2011, Véronique Porra publiait Langue française, langue d'adoption. Une littérature « invitée » entre création, stratégies et contraintes (1946-2000) chez Olms pour proposer le terme « allophones d'expression française » pour se référer à ces « inclassables, convertis à la langue française» (Porra 2011 : 14-15). Dans l'introduction à son ouvrage elle insistait sur le fait que l'intérêt devrait se porter plutôt sur l'explicitation des traits communs reliant un collectif de plus en plus nombreux. Cette thèse nous semble sans doute pertinente et trouve une illustration fort remarquable dans Atlas literario intercultural. Xenografias femeninas en Europa (Alfaro, Mangada 2014) où le florilège d'écrivaines analysées permet de rendre compte de la prépondérance du recours à l'autofiction, au récit de soi comme technique discursive et narrative partagée. Les études proposées mettent en exergue d'une part l'ampleur du corpus (Nancy Huston, Marzena Sowa, Adelaïde Blasquez, Leilla Sebbar entre autres), et d'autre part la proposition d'un nouveau terme pour se référer au collectif croissant de voix au féminin issues du déplacement volontaire ou imposé et qui ont fait de l'écriture en langue française un espace d'expression de l'intime. C'est le cas par exemple de l'écrivaine franco-canadienne d'origine allemande Marguerite Andersen dont l'ensemble de la production littéraire dévoile une écriture au plus près de soi (Melançon 2013). Marguerite Andersen décrira son propre acte d'écriture autobiographique comme " une nécessité. C'est bien pour cela que j'ai, à un moment donné, choisi de faire de ma vie un texte littéraire » (Andersen 1988: 131).

Songeons aussi à L'Année du Cochon de feu de l'écrivaine francophone d'origine vietnamienne, Anna Moï, pour qui l'écriture de soi permet de rendre hommage à la mémoire et à la condition de femme :

Depuis le premier exil vers des territoires plus cléments, fugitive de la guerre à l'âge de dix-sept ans, j'avais toujours recherché l'apaisement dans l'étrangeté ; [...] en me forçant 
Désorientale de Négar Djavadi : exemple d'écriture autofictionnelle comme technique discursive récurrente dans les littératures francophones contemporaines

à des choix quotidiens de mots pour décrire les actes de ma vie, ce journal a été l'occasion d'un zoom sur les zones les plus floues de ma réflexion existentielle. Cet éloge de la lenteur est aussi un hommage à l'âge de femme : à chaque passage des années correspond un gain d'éclairage sur soi-même, ses justesses, ses bavures (Moï 2008 : 375).

Vie et écriture sont intimement liées, de sorte que l'écriture se nourrit de la vie. Estce dire que le recours à son histoire personnelle, à cette composante autobiographique se ferait de façon particulière dans une écriture autofictionnelle?

À cet égard, il nous semble nécessaire préciser que le terme d'autobiographie trouve chez Philippe Lejeune un grand développement. Selon cet auteur, l'autobiographie est un récit rétrospectif en prose qu'une personne réelle fait de sa propre existence, lorsqu'elle met l'accent sur sa vie individuelle, en particulier sur l'histoire de sa personnalité (Lejeune 1996).

En ce qui concerne le terme d'autofiction, celui-ci est un néologisme apparu en 1977, sous la plume de l'écrivain, Serge Doubrovsky qui l'a employé sur la $4^{\text {ème }}$ de couverture de son livre Fils. Il s'agit d'un détournement fictif de l'autobiographie. L'autofiction devient pour certains spécialistes, comme Isabelle Grell, une écriture neuve d'une expérience vécue à la première personne: «L'autofiction, c'est dé-ranger. En disant je. Car en disant je, on dit tu. Tu et moi » (Grell $2014: 108)$.

Le brassage terminologique devient alors nécessairement un sujet de réflexion ( $\mathrm{Si}$ monet-Tenant 2017), soit pour nuancer entre fiction autobiographique et autobiographie autofictionnelle comme le suggérait avec justesse Julia Ori dans son article homonyme (2018), soit pour distinguer entre autobiographie et autobiographique en termes de Louise Dupré pour se référer à toutes ces écritures qui puisent ouvertement dans les données biographiques :

L'autobiographique ne fait pas l'autobiographie. [...] L'autobiographie indique l'appartenance d'une œuvre à un genre littéraire, fondé sur un pacte de vérité entre l'auteur et le lecteur, on le sait. L'autobiographique, pour sa part, renvoie à une matière, à un contenu relatif à l'expérience personnelle que l'auteur cherchera à dissimuler ou, au contraire, à mettre en relief par une stratégie discursive visant à produire des effets référentiels sur le lecteur. Effets qui remettent en question la frontière entre la matière vécue et la matière fictionnelle (Dupré 1996: 73).

Dire son histoire de femme, contribuer à écrire l'Histoire, témoigner de son vécu, ce sont tous des aspects spécifiques qu'Éliane Lecarme-Tabone avait remarqués dans la pratique d'un nouveau genre aux contours incertains et qu'elle désignera comme «écriture de soi au féminin » (2002).

Pour revenir au terme « autofiction » auquel nous adhérons, il semble nécessaire de nous référer de nouveau à Philippe Gasparini quand il déclare :

Si le terme d'autofiction présente un intérêt, pour nous, aujourd'hui, c'est précisément parce qu'il nous permet de désigner l'espace générique dans lequel se 
noue cette nouvelle relation dialectique entre écriture du moi et critique. Et cette vocation essentiellement critique de l'autofiction fut, d'emblée, inscrite dans le processus de son émergence. [...] Dès son apparition, en 1977, en quatrième de couverture d'un « roman » intitulé Fils, le concept d'autofiction s'est construit contre celui d'autobiographie, dans un rapport critique au genre que venait de définir Philippe Lejeune, deux ans auparavant, dans Le pacte autobiographique (Gasparini $2011: 14)$

Cette réflexion fort lucide de Gasparini relance le débat sur la fonction de cet ensemble de productions littéraires contemporaines à la lisière de tous ces genres autour de l'écriture du soi, qui par ailleurs proposent un discours sur la société contemporaine et contribuent à construire l'Histoire comme dans le cas de Elitza Guerguieva (Soto 2019).

Et c'est précisément dans le cadre de ce riche contexte théorique et littéraire que nous aimerions porter notre regard sur la figure de Négar Djavadi, née en Iran en 1969 et partie en exil pour la France à l'âge d'onze ans. C'est son premier roman intitulé Désorientale et paru en $2016 \mathrm{chez}$ Liana Lévi que nous nous proposons d'analyser dans le but d'illustrer le propos évoqué ci-dessus. Force est de constater que le besoin ou le désir de partir, de quitter son pays natal, le déplacement volontaire ou imposé qui s'ensuit nécessairement et l'arrivée ailleurs pour s'intégrer, voire renaître dans bien des cas, apparaissent non seulement comme des vécus communs à la migration, mais aussi comme des catalyseurs créatifs chez de nombreux écrivains étrangers exilés en France, dont bien entendu notre écrivaine.

L'écriture comme création artistique devient ainsi l'occasion de retracer le passé et de remémorer un parcours vital, c'est pourquoi son étude analytique permet d'illustrer la thèse de Coulibaly et Konan (2015) pour qui la littérature en langue française autour de la migration permettrait de transcrire fictionnellement comme des extensions métaphoriques du réel vécu à la première personne le phénomène complexe de la mobilité. L'écrivaine ici convoquée illustre sans doute cette thèse; or, avant d'entamer l'étude de son premier ouvrage, il nous semble nécessaire et pertinent de cerner dans un premier temps son contexte de production.

\section{1. Écrivaines migrantes francophones d'origine iranienne}

Dans le panorama littéraire francophone, les femmes écrivaines d'origine iranienne ont sans doute une place de choix. Songeons à Chahdortt Djavann ${ }^{1}$, immigrée d'origine iranienne, née en 1967 dans une famille aristocratique d'Azerbaïdjan et qui publie un premier roman, Je viens d'ailleurs, paru en 2002 auquel s'ensuivent Comment peut-on être français (2006), Je ne suis pas celle que je suis (2011) et La dernière séance (2013). L'écriture devient pour elle, de même que pour ses compatriotes, l'occasion de parti-

$1 \quad$ Elle quitte l'Iran en 1991 à cause du régime politique islamique. Elle s'installe en Turquie où elle travaille et étudie pendant deux ans, puis arrive à Paris en 1993 sans savoir parler français. Elle s'initie à l'apprentissage de la langue française puis entame des études en anthropologie à l'EHESS. 
Désorientale de Négar Djavadi : exemple d'écriture autofictionnelle comme technique discursive récurrente dans les littératures francophones contemporaines

ciper au débat politique sur des sujets sensibles et polémiques qui traversent la société française et qui sont directement ou indirectement liés à l'immigration, comme le port du voile.

Un autre nom sans doute célèbre de cette diaspora iranienne est le cas de Marjane Satrapi, auteure de Persépolis, son autobiographie en bande dessinée. Outre Djavann et Satrapi $^{2}$, on mentionnera Fariba Hachtroudi ${ }^{3}$, Sorour Kasmaï 4 , Sara Yald ${ }^{5}$, Delphine Minoui ${ }^{6}$, Abnousse Shalmani ${ }^{7}$ et plus récemment Maryam Madjidi (France, 1980), auteure de Marx et la Poupée, Goncourt du premier roman 2017.

Leurs contributions littéraires s'inscrivent dans la littérature d'exil ou littérature migrante francophone, récits qui témoignent d'expériences d'exil, de ses causes et de ses conséquences, au croisement de la petite histoire personnelle et de la Grande Histoire. À partir de leur récit de vie raconté selon des modalités différentes de l'écriture de soi, ces auteures témoignent les événements traumatiques qui bouleversent la vie individuelle et collective.

\section{Désorientale de Négar Djavadi}

Dans le cas qui nous occupe, force est de constater l'empreinte indélébile du contexte historique et politique dans l'écriture de soi qui jalonne le premier texte narratif de Négar Djavadi. C'est en parcourant sa trajectoire vitale que nous repérons de nombreuses traces autobiographiques sous-jacentes à la construction du récit. En effet, les ressemblances sont importantes, par exemple entre le père de Kimiâ Sadr, narratrice et personnage principal du roman Desorientale et le père de Négar Djavadi, puisque tous les deux furent condamnés à mort en Iran au début des années quatre-vingt. Ils travaillaient tous les deux comme journalistes à Téhéran militant dans l'opposition et appartenaient à la haute bourgeoisie francophile du pays. Au cours des années soixante, ils oseront écrire contre le Shah, ce qui aussi bien dans la fiction que dans la réalité, leur vaudra d'être persécutés par la SAVAK, le service secret d'intelligence iranienne. On interdira à leurs femmes d'enseigner, puis seront arrêtés à maintes reprises. Après la chute du Shah, leur décision de se manifester

2 Née en Iran en 1969 dans le cadre d'une famille aisée, elle est envoyée à Vienne pour poursuivre ses études de Secondaire. Son œuvre, fort remarquée par la critique est devenue un symbole de tolérance et de liberté.

3 Née à Téhéran en 1951, Fariba Hachtroudi est une journaliste et écrivaine franco-iranienne. Un de ses ouvrages les plus renommés est À mon retour d'Iran (2008) où l'empreinte du vécu autobiographique est une composante essentielle, de même que la trajectoire littéraire de ses compatriotes.

4 Sorour Kasmaï naît à Téhéran en 1962. Romancière, traductride et éditrice, elle est l'auteure de trois romans, dont La vallée des aigles (2006), prix littéraire de l'Asie où la narratrice éloge les femmes iraniennes qui ont résité la tyrannie.

5 Née en Iran en 1967, cette journaliste francophone d'origine iranienne a publié un premier roman intitulé Regard persan en 2007.

6 Journaliste et écrivaine française d'origine iranienne, Delphine Minoui (1974) est spécialiste du Moyen Orient. Seule femme journaliste de langue française en Iran pour la presse française, elle reçoit le Prix Albert Londres de Journalisme en 2006. Auteure de plusieurs ouvrages, nous retiendrons Je vous écris de Téhéran de 2015.

$7 \quad$ Née à Téhéran en 1977, ses parents fuient l'Iran et se réfugient en France en 1985. Journaliste et réalisatrice, elle publie un premier roman en 2018, intitulé Les exiles meurent aussi d'amour. 
publiquement contre l'Imam Ayatollah Khomeiny, provoquera la colère du dirigeant fondamentaliste qui en 1981 décrétera une «fatwa», une peine de mort, contre le père de Négar Djavadi ce qui trouvera une transposition dans la fiction. C'est alors que la fuite de l'Iran vers la France s'impose et commence ainsi l'exil aussi bien pour Négar Djavadi que pour la protagoniste du récit.

Diplômée de l'INSAS, une école de cinéma bruxelloise, Négar Djavadi travaille quelques années derrière la caméra, pour devenir scénariste, aussi bien de documentaires que de séries. Actuellement elle vit à Paris, où elle a écrit et publié un premier roman en français, bien accueilli par la critique. Le prix littéraire de la Porte Dorée 2017 lui est attribué, un prix qui récompense chaque année une œuvre de fiction écrite en français ayant pour thème l'exil, l'immigration, les identités plurielles ou l'altérité liée aux réalités migratoires. Désorientale se lit comme un creuset de représentations de la migrance qui se démultiplient dans chaque axe structural du roman. Il sera donc question de cicatrices dans la langue, des paysages de l'enfance, de l'Iran des années 70, mais bien entendu du mythe du départ et du drame de l'exil. Une constellation thématique où s'imbrique exil/identité/appartenance/mémoire et qui prend une configuration spécifique où la langue française joue un rôle axial.

Ainsi, et dans le but de répondre au titre de notre article, notre étude s'attardera dans un premier temps à analyser la structure du récit en parallèle avec la chronologie et la spatialité, l'objectif étant de montrer jusqu'à quel point le départ imposé va favoriser une structure dichotomique entre l'évocation incessante et inévitable de l'Iran de l'enfance et la narration de la nouvelle vie en exil. De ce fait, la temporalité va se polariser nécessairement entre le passé évoqué aux caprices des souvenirs et un temps présent qui ne peut être compris sans les clés de cette remémoration. Au même temps, la coordonnée spatiale prendra forme spéculaire où vont se refléter les deux univers auxquels seront condamnés d'appartenir les protagonistes de l'histoire: d'une part la ville de Téhéran, la mer Caspienne et le village de Mazandaran, dans le nord du pays et, d'autre part, le Paris mythique qui ne tardera pas à devenir un espace de souffrance, de solitude, de douleur et qui obligera les personnages à se confronter à eux-mêmes.

\subsection{Deux faces pour une structure bipolaire en rapport avec l'entre-deux}

La lecture du premier roman de Négar Djavadi nous mène à nous attarder dans un premier temps sur le choix porté par l'écrivaine de construire son premier roman en deux faces comme les vieux disques de vinyle. Un clin d'œil musical qui doit d'ailleurs se lire en clé autobiographique puisque c'est Négar Djavadi qui explique dans une interview réalisée par Zoé Varier dans le programme de France Inter D'ici, d'ailleurs, que la musique et plus particulièrement le piano, devint son refuge lors de son arrivée abrupte en France après avoir traversé à pied et à cheval la frontière turco-iranienne dans le Kourdistan. Cette dimension thématique percera élégamment le texte avec maintes références intermusicales. N'oublions pas par ailleurs de remarquer la présence habituelle de références au cinéma sous forme de citations d'acteurs ou de films, mais aussi à travers des constructions descriptives qui dévoilent le regard précis et sensible de la cinéaste Négar Djavadi. 
En revenant à la structure de ce roman, nous pouvons constater que dès les premières pages, le lecteur comprend rapidement que la structure narrative va se construire autour d'une duplicité réitérative. Assise dans une salle d'attente d'un hôpital consacré à la reproduction assistée, Kimiâ Sadr, la narratrice et protagoniste profite de cette attente pour retracer et reconstruire suivant au gré des souvenirs, la saga familiale des Sadr sur quatre générations. Ainsi, deux narrations s'enchevêtrent et avancent parallèlement bien qu'avec un rythme, une chronologie et une topographie spéficique à chaque pan de la narration. Le profil de Kimiâ se construira au fil des dix chapitres de la première partie du récit, bien que ce n'est qu'au chapitre sept intitulé « Le destin selon Grand-Mère » que le lecteur apprend le sort du destin qui voulut que Kimiâ naisse le même jour de la mort de sa grand-mère Nour. Ses parents désiraient un garçon après avoir eu deux filles, mais la mort de Nour conditionna le résultat final. L'enfant qui devait naître un garçon, naquit finalement un fille, tel que l'avait prédit et compris tendrement la grand-mère maternelle, Emma Aslanian, une arménienne qui savait lire dans le marc du café. Ce personnage attachant rédigera plus tard une lettre à sa fille pour lui prier d'être compréhensive envers sa petite-fille, elle, qui avait si bien compris que le destin de Kimiâ était nécessairement uni à cette dualité homme-femme qui se manifestera forcément dans ses tendances sexuelles. Cette insertion dans le récit d'une autre voix narrative rend polyphonique un récit qu'elle s'est imposé de d'écrire, car : « Aucun ne prendrait la peine de comprendre, de poser des questions, de me regarder, moi aussi, comme une somme incongrue de circonstances, de fatalité, d'héritages, de malchances et de drames. C'est pourquoi j'écris » (Djavadi $2016: 19$ ).

Les désirs de maternité chez Kimiâ et les péripéties pour devenir mère percent le texte de nombreuses réflexions sur la condition et le destin des femmes en Iran. L'apparition dès les premières pages de Pierre, son compagnon apparent, obligent le lecteur à se construire une image de Kimiâ qui pourtant chavirera dans la deuxième face/partie du récit. En effet, après la révélation de Leïli, sa sœur ainée au dernier chapitre de la première partie, le lecteur est confronté, de même que la protagoniste à sa véritable nature sexuelle, ainsi nous lisons: "Vous me direz : c'est cliché l'histoire de cette fille dont le père veut un fils, qui devient un garçon manqué et finit lesbienne. C'est vrai » (Djavadi 2016 : 144). Cette révélation de Leilli procurera un virement dans la structure du récit, de sorte qu'à partir de la deuxième partie de Désorientale, la saga familiale étant déjà présentée, c'est le récit du départ pour la France qui prend la relève, suivi de l'évocation poignante et sincère des difficultés de l'arrivée, l'impossibilité de l'intégration pour Darius et Sara et les tentatives des trois sœurs de s'intégrer comme première génération d’immigrantes débarquées à Paris.

\subsection{La révolution iranienne vs le Paris des années $\mathbf{9 0}$, deux axes chronologiques autour d'une même vie}

Le double espace - salle d'attente vs L'Iran familial constamment remémoré - va forcément déclencher une double temporalité où aux minutes interminables à la salle d'attente viennent se confronter des années et même des siècles écoulés dans la recons- 
truction de la saga familiale avec comme toile de fond, le contexte historique de la Révolution iranienne. La grande Histoire vient ainsi encadrer les petites histoires de chaque personnage de cette immense saga évoquée, de sorte que ces mêmes personnages vont également se dédoubler: au passé appartiendront les grands hommes et les grandes femmes de la famille Sadr, alors qu'au présent appartiennent la mère, Sara et les sœurs.

En ce sens, nous observons que la chronologie du récit repose sur un équilibre constant réussi grâce à des allées et des retours entre le passé et le présent de la vie de la narratrice où vont venir s'enchevêtrer le reste des membres de la saga familiale des Sadr.

La lecture du roman offre au lecteur des références bien précises à des événements appartenant au contexte historique qui seront méticuleusement datés, permettant ainsi de dresser un axe temporel qui portera le lecteur de 1978 jusqu'en 2001. C'est le cas très particulièrement de ce que la narratrice nommera «L'EVENEMENT » pour se référer, et cela le lecteur ne l'apprendra qu'au quatrième chapitre de la deuxième partie, à l'assassinat de son père le 11 mars 1994. Pourtant, dans la salle d'attente, le temps semble ne pas vouloir avancer. Soumise dans ses souvenirs qui permettent à la narratrice de retracer l'histoire familiale, Kimiâ attend pendant un temps indéfini que le Docteur Gauthier veuille bien arriver et procéder à l'insémination, mais aucune référence temporelle n'est fournie et ce n'est que vers la fin du récit que le lecteur découvre comment Kimiâ a fini dans cette salle toute seule et pourquoi Pierre n'est pas son compagnon, mais un autre personnage appelé Anna. Au bout des dix chapitres de la première partie, on constate que le temps écoulé couvre à peine deux heures, alors qu'entre temps, dans la pensée de Kimiâ quatre générations d'une même famille ont été dépeintes parallèlement. Il en résulte une disrupture temporelle qui avait été ainsi annoncée par la narratrice, devenue alter-ego fictionnel de l'écrivaine et qui manifeste ouvertement sa prise de position à l'égard d'une écriture autofictionnelle :

Raconter le présent exige que je remonte loin dans le passé, que je traverse les frontières, survole les montagnes et rejoigne ce lac immense qu'on appelle mer, guidée par le flux des images, des associations libres, des soubresauts organiques, les creux et les bosses sculptés dans mes souvenirs par le temps. Mais la vérité de la mémoire est singulière n'est-ce pas ? La mémoire sélectionne, élimine, exagère, minimise, glorifie, dénigre. Elle façonne sa propre vision des événements, livre sa propre réalité. Hétérogène mais cohérente. Imparfaite, mais sincère (Djavadi 2016 : 10-11)

\subsection{La topographie de l'exil, du Paris mythique aux souvenirs nostalgiques de l'Iran lointain}

Cette dualité temporelle aux contrastes si opposés, nous la retrouvons de nouveau projetée dans la dimension spatiale. En effet, la salle d'attente fait l'objet de descriptions sobres perçues à travers le regard de la narratrice qui attend son tour avec résignation. Ce sont les pensées de Kimiâ qui l'emportent et de manière très révélatrice l'évocation des espaces de l'enfance. Téhéran apparaît comme espace bruyant et pas toujours très propre ; les références aux saisons complètent la fresque de cette grande ville persane située dans 
Désorientale de Négar Djavadi : exemple d'écriture autofictionnelle comme technique discursive récurrente dans les littératures francophones contemporaines

une vallée entourée de montagnes enneigées. Mais ce sera dans l'exil où l'ampleur du sentiment d'appartenance à cet espace se multipliera. L'école, l'appartement, l'épicerie d'Agha Mohabati deviendront des endroits rassurants et familiers. L'Iran, pour Kimiâ sera souvent évoqué à travers les nombreuses références à la mer Caspienne et plus particulièrement à Mazandaran, région du nord de l'Iran où une partie de la saga Sadr habite et où Kimiâ passera les étés. Tout y décrit, le vert des arbres, la fraicheur de la pluie, son vent qui l'attrape et la rattache à ses origines. C'est à Mazandaran qu'elle découvrira sa véritable essence et c'est là que se trouve l'andarouni, cet espace maintes fois évoqué, qui représente un bâtiment exclusivement réservé aux femmes et si caractéristique de l'architecture persane. Synonyme d'intimité et de liberté pour les femmes, les grands hommes Sadr redouteront l'andarouni par son manque de contrôle.

Face à la sécurité de ces lieux connus, dans l'imaginaire enfantin de Kimiâ, Paris et par extension la France, apparaît comme un endroit mythique, toujours propre, cultivé et élégant. Songeons très particulièrement au passage suivant :

Sara nous emmenait dans un supermarché français ouvert dans une des rues huppées du nord de la ville. D'une propreté intimidante, l'endroit était rempli de toute sorte de marchandises qui nous paraissaient terriblement exotiques. Des petits bouts de France, détachés d'une totalité aussi inaccessible qu'un rêve, qui miracle du pétrole, avaient voyagé jusqu'à nous. Vache qui rit, Nutella, yaourts Danone, camembert Caprice des Dieux, savon Zeste, cigarettes Gitane. Les produits aux emballages brillants étaient rangés sur des rayonnements en fer, offerts à la vue, disponibles, et non entassés en une montagne branlante comme chez notre épicier, Agha Mohabati (Djavadi 2016 : 35).

Pourtant, une fois arrivée à Paris, Kimiâ raconte avec rudesse la vraie signification que va acquérir cette terre d'accueil. La France de ses illusions devient un espace anonyme, hostile où elle se laisse aller faute de l'impossibilité à s'intégrer, " cette ville, autrefois vénérée pour sa beauté, devenue froide et hostile» (Djavadi 2016 : 279). Nous lisons également: «Car pour s'intégrer à une culture, il faut, je vous le certifie, se désintégrer d'abord, du moins partiellement, de la sienne» (Djavadi 2016 : 114). Le lecteur assiste alors au récit d'une adolescence difficile et la rupture par rapport à l'image offerte jusqu'à présent de Kimiâ. Et c'est avec une fine sensibilité que la narratrice montre au lecteur dans le deuxième chapitre de la deuxième partie, intitulé de manière épiphanique « Terre promise, pays perdu » comment chaque membre de sa famille va expérimenter son exil. Nous lirons alors: «Le déracinement avait fait de nous, non seulement des étrangers chez les autres, mais des étrangers les uns pour les autres » (Djavadi 2016 : 273). Le titre du roman retrouve alors dans le récit du départ en exil toute sa signification: « Bientôt, mon prénom ne sera plus prononcé de la même manière, le «â» final deviendra «a» dans les bouches occidentales, se fermant pour toujours. Bientôt, je serai une «désorientale» (Djavadi $2016: 257$ ).

Dans cette dichotomie constante où bascule constamment le texte, les deux personnages les plus emblématiques du récit apparaissent une et mille fois tout au long 
de la narration sous regards kaléidoscopiques, nous rapprochant des aspects les plus intimes, nous montrant leur véritable essence et manifestant le grand amour que ressent la protagoniste envers ses parents. Sara et Darius, Darius et Sara, un grand couple qui réveillera l'admiration de Kimiâ et qui ne pourra s'empêcher de les évoquer constamment pour nous découvrir leur essence, mais aussi leur faiblesse et leur souffrance. Sara, la mère apparait avec détermination au deuxième chapitre de la première partie et fera l'objet de belles descriptions. Intelligente, sensible, compromise, elle épaulera son mari, le suivra partout et finira par perdre la raison, une fois son mari assassiné. La dernière image que nous offre Kimiâ d'elle, nous montre une femme qui a perdu toute sa vitalité, assise immobile dans une chambre d'une résidence à attendre indéfiniment l'arrivée de son mari déjà décédé. Quant à lui, le grand Darius aux yeux bleus, exerce une grande admiration chez la jeune Kimiâ ; ce journaliste intrépide qui n'avait pas peur de faire parler sa plume finira pourtant ses jours à Paris, exilé, condamné au silence, à errer dans la ville, tout seul durant des heures et des heures. Sa mort fatidique aura un effet perturbateur chez sa femme et ses trois filles. Une mort qui symbolise la perte de tout un témoignage d'un intense vécu d'où l'insistance de Sara de recueillir toute cette vie dans un récit.

C'est donc pour cela et dans le but de ne pas oublier que Sara entreprendra déjà en Iran, l'écriture de leur récit de vie : « Darius démissionna du journal Keyhan le 13 novembre 1975. C'est par cette date que Sara commen Notre vie, le livre-témoignage écrit dans la solitude parisienne et relatant les années de Révolution jusqu'à notre départ pour la France » (Djavadi 2016 : 173). Dans la fiction, ce texte aura en Iran un grand succès, d'où le désir de Sara d'être traduite par ses filles. La charnière entre fiction et réalité devient floue de nouveau et situe le lecteur dans les marges de cette écriture de soi dont parlait Éliane Lecarme-Tabone (2002) ; voire une écriture fictionnelle d'une expérience de vie à partager. Par ailleurs, l'insertion dans la narration de ce nouveau cadre énonciatif enrichit la dimension polyphonique du récit où s'entremêlent la narration de Kimiâ aux extraits du récit de Sara, décrite par sa fille comme « la mémoire de ce lieu, la gardienne de leur histoire » (Djavadi 2016 : 245). Quelques bribes de ce manuscrit tomberont dans les mains de Kimiâ qui les intégrera élégamment dans sa narration :

Leili a trouvé ce texte inachevé, écrit à la main en français sur des feuilles quadrillées, dans les affaires de Sara. [...] Come je vous l'ai dit, je n'ai jamais eu le courage de lire le livre de Sara jusqu'au bout, mais selon Mina ce texte est une traduction fidèle du passage concernant notre départ. [...] Sans le dire clairement, Sara aurait aimé que l'une de nous traduise son livre en français (Djavadi $2016: 243$ ).

C'est grâce à l'histoire de Sara que le lecteur peut imaginer par exemple la difficile traversé du Kourdistan pour quitter l'Iran, mais surtout comprendre sous un autre regard qu'elles étaient «étrangères chez nous» (Djavadi 2016 : 238). Une fois accepté son sort, Kimiâ décidera d'entreprendre l'écriture de son histoire, de l'histoire de son père, de l'histoire racontée par sa mère en persan et qui tenait tellement à être traduite en français, pour ne pas oublier, dira-t-elle (Djavadi 2016 : 243). Transférer le vécu, la 
Désorientale de Négar Djavadi : exemple d'écriture autofictionnelle comme technique discursive récurrente dans les littératures francophones contemporaines

légende familiale, faire revivre le passé, car c'était bien Darius qui lui avait dit à Kimiâ : « Si tu as quelque chose à dire, écris-le » (Djavadi $2016: 19$ ).

L'écriture de cette grande saga devient alors un hommage, un chant à la famille, comme cellule ancestrale. L'importance des liens familiaux, l'admiration et la reconnaissance envers l'autre percent et parcourent le texte en tant que thématiques axiales. Le récit s'ouvrait avec Kimiâ suivant son père à Paris et il se referme avec une réconciliation poignante entre Kimiâ et sa mère, lui priant d'avoir des enfants pour que la tradition de la grande saga des Sadr perdure. Nous apprenons alors que Kimiâ est enceinte, et la boucle peut être ainsi bouclée.

\section{Autofiction, autobiographie et écriture au plus près de soi pour ne pas oublier}

L'approche analytique du premier roman de Négar Djavadi dévoile un goût manifeste pour l'autofiction où l'écriture de soi est à la base de la création. Écrire pour ne pas oublier avait suggéré Paul Ricœur (1999). Pour le philosophe, dans le rapport entre temps vécu et les configurations narratives du récit la médiation de la mémoire joue un rôle essentiel, car, « uno no recuerda solo, sino con ayuda de los recuerdos de otro » (Ricœur 1999: 17).

À cet égard nous pouvons affirmer que la remémoration du passé et l'écriture de la grande Histoire pour configurer la mémoire collective convoque nécessairement la commémoration et la mémoire individuelle. C'est ainsi que l'exprimait l'historien canadien Gaétan Gervais :

L'identité d'une communauté se fonde sur une certaine mémoire de son passé, sur une certaine compréhension historique. [...] L'expérience historique résulte des événements réels mais le souvenir qu'on en garde se prête bien à des interprétations. La part du subjectif est donc importante dans la définition d'une identité, les faits et les événements étant sans cesse réinterprétés par les communautés (Gervais 1995 :166-167).

Les traces d'une mémoire blessée percent le texte des écrivaines citées au cours de ces pages. Chez Djavadi, mais aussi chez Moï ou Andersen, le témoignage historique et les traces du vécu de la mémoire réclamaient d'un genre discursif qui puisse s'énoncer à la première personne. La mémoire individuelle par sa valeur de témoignage vient enrichir la mémoire collective et c'est justement dans cet interstice que se situe l'écriture de Négar Djavadi ainsi que ses compatriotes qui ont pris la plume pour dire.

Or, outre les brouillages entre fiction, réalité et mémoire qui caractérisent cette écriture au plus près de soi, c'est la valeur autobiographique de ces témoignages exotiques qui déclenchent l'intérêt chez le lecteur :

D'aucuns choisiront de consacrer leur œuvre à leur pays d'origine, par nostalgie, parce que c'est ce qu'ils maitrisent le mieux, peut-être aussi parce que c'est ce que l'on attend d'eux dans une certaine perspective exotique; d'autres évoqueront leur expérience 
de l'exil et, ce faisant, leur expérience du pays d'accueil et y verront une façon de formuler, de verbaliser leur écartèlement voire le mal-être dans l'expérience de l'écriture du passage et de la recherche identitaire; d'autres enfin, plus rarement, chercheront à sortir de cette dialectique origine/entre-deux et consacreront leurs romans à de tout autres problèmes, se refusant à l'écriture identitaire, à l'explication, à la problématisation de leur situation identitaire au niveau littéraire (Porra 2011 : 79-80).

En tout cas, le roman autofictionnel devient l'acte de communication par excellence par sa potentialité, par les hybridations qu'il rend possible, para la multiplicité de voix qu'il assure. La migration, chez nos écrivaines, devenue forcément exil, rend possible la création pour témoigner mais aussi pour s'assurer et retrouver une représentation identitaire.

\section{BIBLIOGRAPHIE}

ALFARO, Margarita y MANGADA, Beatriz (coord.) (2014) : Atlas literario intercultural. Xenografias femeninas en Europa. Madrid : Calambur.

ANDERSEN, Marguerite (1988) : «L'autobiographie: tentation ou nécessité? », Morency, Pierre (ed.), La tentation autobiographique. Montreal : L'Hexagone : 131-132.

COULIBALY Adama, KONAN Yao Louis (dir.) (2015) : Les Écritures migrantes. De l'exil à la migrance littéraire dans le roman francophone. Paris : L'Harmattan.

DELBART, Anne-Rosine (2005) : Les exilés du langage. Un siècle d'écrivains français venus d'ailleurs (1919-2000). Limoges: Presses de l'Université de Limoges.

DJAVADI, Négar (2016) : Désorientale. Paris : Liana Levi.

DUPRÉ, Louise (1996) : "Le lièvre de mars de Louise Warren. Vers une 'réalité virtuelle' », Voix et Images, 64-1 : 63-77.

GASPARINI, Philippe (2011) : «Autofiction et autobiographie », Tangence, 97 : 11-24.

GERVAIS, Gaétan (1995) : " Aux origines de l'identité franco-ontarienne », Cahiers Charlevoix. Études franco-ontariennes, $1:$ 125-168.

GRELL, Isabelle (2014) : L'Autofiction. Paris: Armand Colin.

LECARME-TABONE, Éliane (2002) : «Existe-t-il une autobiographie des femmes? », Magazine littéraire. Les écritures du moi, de l'autobiographie à l'autofiction, $409: 56-59$.

MATHIS MOSER, Ursula, MERTZ-BAUMGARTNER, Birgitz (2012) : Passages et ancrages. Dictionnaires des écrivains migrants de la langue française (19812011). Paris : Honoré Champion.

MELANÇON, Johanne (dir.) (2013): L'écriture au féminin au Canada français. Sudbury : Prise de Parole.

MOÏ, Anna (2008) : L'année du cochon de Feu. Monaco : Éditions du Rocher.

ORI, Julia (2018) : «Ficción autobiográfica y autobiografía ficcional : La busca del jardín y Ce que la nuit raconte au jour de Héctor Bianciotti », Anales de Filología Francesa, $26: 479-502$. 
Désorientale de Négar Djavadi : exemple d'écriture autofictionnelle comme technique discursive récurrente dans les littératures francophones contemporaines

PORRA, Véronique (2011) : Langue française, langue d'adoption. Une littérature « invitée », entre création, stratégies et contraintes (1946-2000). Hildesheum, Zürich, New York : Olms.

RICCEUR, Paul (1999) : La lectura del tiempo pasado : memoria y olvido. Madrid: UAM Ediciones / Arrecife.

SIMONET-TENANT, Françoise (dir.) (2017): Dictionnaire de l'autobiographie. Écritures de soi de langue française. Paris: Honoré Champion.

SOTO, Ana Belén (2019) : " Elitza Gueorguieva : identité, témoignage et écriture », Çédille, revista de estudios franceses, 15: 533-553.

VARIER, Zoé (2016) : Interview à Négar Djavadi sur France Inter [https://www. franceinter.fr/emissions/d-ici-d-ailleurs/d-ici-d-ailleurs-12-novembre-2016; $12 / 05 / 19]$

\section{PERFIL ACADÉMICO Y PROFESIONAL}

Beatriz Mangada es Profesora Contratada Doctora de la Universidad Autónoma de Madrid. Desde la defensa de su tesis doctoral, que versó sobre literatura franco-canadiense, ha centrado su investigación en torno las literaturas francófonas, de manera particular en las producciones literarias en femenino marcadas por el desplazamiento. Trabaja igualmente en el ámbito de la didáctica del francés como lengua extranjera y la adquisición de la competencia intercultural.

Es miembro del grupo de investigación ELITE (HUM-F065), Estudio de las Literaturas Transnacionales en Europa.

Fecha de recepción: 16/05/2019

Fecha de aceptación: 13/07/2019 